\title{
LAS ELECCIONES DOMINICANAS DEL AÑO 2000 \\ EN EL CONTEXTO DE LA HISTORIA ELECTORAL NACIONAL
}

Víctor Ruíz*

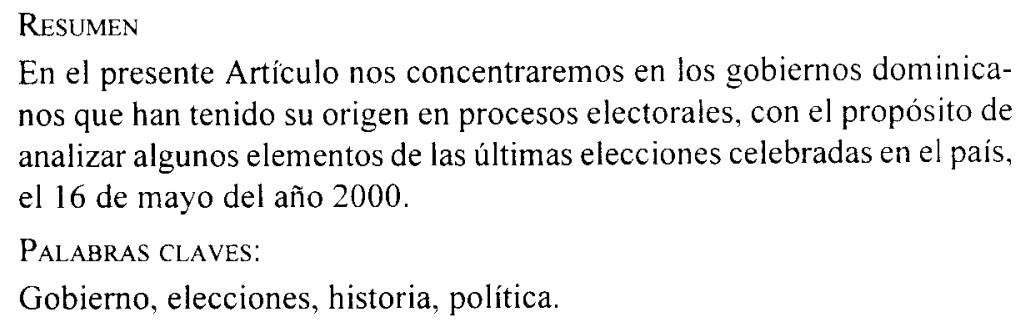

La República Dominicana tiene una dilatada experiencia en cambio de gobiernos. Desde 1844 hasta la fecha, estudios que hemos realizado nos permiten afirmar que en el país ha habido unos 143 gobiernos.

Se trata de una cifra alta si se relaciona con los 156 años de historia como nación del pueblo Dominicano. El balance pone de manifiesto que el país ha tenido marcada inestabilidad política. Esta situación fue indudablemente más acusada durante el siglo XIX que durante el XX. De los 143 gobiernos que se han formado en el país, 86 de ellos se originaron en dicho siglo, en un

\footnotetext{
(-) Universidad INTEC

Ruiz Burgos. Victor Eddy. 1993. Los gobiernos de la Repriblica Dominicana. Santo Domingo. Biblioteca Nacional.
} 
período de 55 años. En términos relativos esto significa que cerca del $60 \%$ de los gobiernos registrados en la República Dominicana hasta el año del 2000 se constituyeron en el siglo XIX.

Si se considera que cuando hablamos del siglo XIX nos referimos al período de 55 años que va desde 1844 hasta 1899 , resalta un dato relevante: durante el lapso en cuestión en el país se creó, en términos relativos, un gobierno cada 6 meses aproximadamente, lo que evidencia la inestabilidad política ya aludida.

Tantos gobiernos se han originado de maneras diferentes. Así, buscando patrones podríamos clasificar el origen de los gobiernos dominicanos de acuerdo a la siguiente tipología:

\begin{tabular}{|c|c|}
\hline Elecciones ..... & 41 \\
\hline Revoluciones .............. & 28 \\
\hline 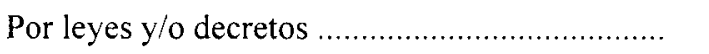 & 8 \\
\hline Designación por grupos de poder local ............. & 25 \\
\hline 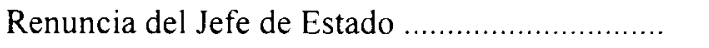 & 12 \\
\hline 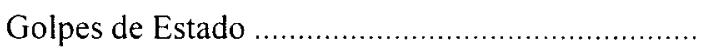 & 9 \\
\hline 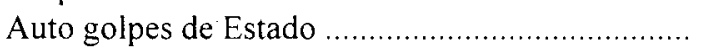 & 7 \\
\hline 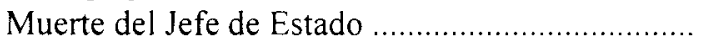 & 4 \\
\hline Otros & 9 \\
\hline TOTAL $\ldots \ldots \ldots \ldots \ldots \ldots \ldots \ldots \ldots \ldots \ldots$ & 143 \\
\hline
\end{tabular}

En el presente artículo nos concentraremos en aquellos gobiernos cuyo origen ha sido las elecciones, con el propósito de analizar algunos elementos del último certamen electoral celebrado en el país el 16 de mayo del año 2000.

\section{Las elecciones en la historia política de la República Dominicana}

Las elecciones han tenido un importante papel en el origen de los gobiernos dominicanos ${ }^{2}$. Estas han sido la forma en que

2 Ver la otra clave de Julio Genaro Campillo Pérez Historia Electoral Dominicana. 1986. Santo Domingo, Cuarta edición 
los dominicanos han formado mayor número de gobiernos, como lo evidencia el cuadro precedente. En términos absolutos en el país se han celebrado 41 torneos nacionales a lo largo de su historia, incluyendo el del 16 de mayo del año 2000.

¿Cuándo se originan las elecciones en la República Dominicana?

Desde que fue fundado el país se contempló constitucionalmente la selección de sus autoridades nacionales por la vía electoral ${ }^{3}$. Empero, los primeros gobiernos nacionales surgieron de manera no constitucional. Estos fueron juntas gubernativas de corta duración.

La República Dominicana hizo su debut político al escindirse de la nación haitiana, pero no surgió como un proyecto socialmente coherente ${ }^{4}$. Elementos sociales con dos posturas diferentes pactaron a fin de lograr la ruptura de Haití. Un grupo abogaba por la creación de un estado dominicano, completamente libre e independiente; el otro grupo, empero, deseaba tan solo la separación de Haití, para anexar el país a Francia. Finalmente estos últimos se impusieron sobre sus rivales ${ }^{5}$. No obstante, para alcanzar su victoria tuvieron que ejercer la violencia contra sus opositores, desencadenándose luchas que se manifestaron en el plano político a través de las aludidas juntas centrales gubernativas.

El primer gobierno constitucional dominicano fue precisamente la cristalización del triunfo de la facción anexionista, de ahí que no fuera el producto, precisamente, de torneo electoral alguno. Fue en el sentido estricto de la palabra una designación

\footnotetext{
3 Peña Battle, Manuel Arturo (Apuntes y compilación). 1944. Constitución Politica y Reformas Constitucionales 1844-1942. 2 tomos. Santiago, Gobierno Dominicano.

+ Rodriguez Demorizi, Emilio. 1982. Papeles del general Santana. Santo Domingo. Fundación Emilio Rodríguez Demorizi.

Rodríguez Demorizi, Emilio. 1956. Correspondencia del Cónsul de Francia en Santo Domingo, 1844-1846, 2 vols. Ciudad Trujillo. editora Montalvo.
} 
por el grupo de poder triunfante, que tras instaurar un congreso constituyente y aprobar la primera constitución del estado dominicano, designaron al general Pedro Santana como presidente del país, en virtud del artículo 205 de la carta sustantiva. Para completar este hecho, en el artículo 206 se estableció que por esa sola oportunidad el presidente seleccionado tendría dos mandatos consecutivos de cuatro años cada uno. En consecuencia el segundo de estos mandatos concluirá el 15 de febrero de 1852. Para ampliar de forma casi total todos los poderes del presidente, los constituyentes de 1844 le agregaron a la carta magna el artículo 210 , que le otorgaba a éste poderes ilimitados mientras persistiera el estado de guerra en el país. Así, en la constitución de noviembre de 1844 se encuentran las raíces de las elecciones dominicanas.

A pesar de tal concentración de poderes, Santana gobernó solamente por cuatro años, al cabo de los cuales se vio en la obligación de renunciar, dándole paso así a las primeras elecciones celebradas en el país en el año de 1848. No nos detendremos a analizar los pormenores de ésta ni las siguientes elecciones, ya que no es nuestro interés en esta oportunidad.

\section{Tipo de elecciones contempladas.}

Desde 1844 se ha contemplado en el país que las elecciones se realicen utilizando dos tipos diferentes de votos: el indirecto y el directo. El primero, mediante representación de electores y el otro por una participación masiva de la población. Las elecciones con votos directos han prevalecido sobre las que han utilizado votos indirectos. De las primeras se han celebrado 27 hasta la fecha y de las segundas 15 .

\section{El contexto electoral.}

Independientemente del tipo de votos, las elecciones dominicanas se han celebrado en tres contextos básicos, lo que permite 


\begin{tabular}{|llrl|}
\hline \multicolumn{4}{|c}{ ELECCIONES ELECTORALES NACIONALES 1848-2000 } \\
\multicolumn{4}{|c|}{ POR VOTOS DIRECTOS } \\
Año & Candidato triunfante & Votos & Principal opositor \\
1866 & José María Cabral & 4,389 & Otros candidatos \\
1874 & Ignacio M. González & 15,533 & Manuel A. Cáceres \\
1876 & Ulises F. Espaillat & 24,329 & Otros candidatos \\
1878 & Ignacio M. González & 6,203 & G. Luperón \\
1878 & Cesáreo Guillermo & 18,344 & Otros candidatos \\
1880 & Fernando A. De Meriño & 17,398 & Otros candidatos \\
1882 & Ulises Heureaux & 23,036 & Otros candidatos \\
1884 & Francisco G. Billini & 34,951 & Segundo Imbert \\
1886 & Ulises Heureaux & 43,740 & Casimiro de Moya \\
1930 & Rafael L. Trujillo & 223,926 & Votos en contra \\
1934 & Rafael L. Trujillo & 256,423 & Ningún opositor \\
1938 & Jacinto B. Peynado & 319,680 & Ningún opositor \\
1942 & Rafael L. Trujillo & 581,937 & Ningún opositor \\
1947 & Rafael L. Trujillo & 781,389 & Otros candidatos \\
1952 & Héctor B. Trujillo & $1,038,816$ & Ningún opositor \\
1957 & Héctor B. Trujillo & $1,265,681$ & Ningún opositor \\
1962 & Juan Bosch & 628,044 & Viriato Fiallo \\
1966 & Joaquín Balaguer & 769,265 & Juan Bosch \\
1970 & Joaquín Balaguer & 653,565 & Francisco A. Lora \\
1974 & Joaquín Balaguer & 942,726 & L. H. Lajara Burgos \\
1978 & S. Antonio Guzmán & 856,084 & Joaquín Balaguer \\
1982 & S. Jorge Blanco & 854,868 & Joaquín Balaguer \\
1986 & Joaquín Balaguer & 874,453 & Jacobo Majluta \\
1990 & Joaquín Balaguer & & Juan Bosch \\
1994 & Joaquín Balaguer & & J. F. Peña Gómez \\
1996 & Leonel Fernández & & J. F. Peña Gómez \\
2000 & Hipólito Mejía & & Danilo Medina \\
\end{tabular}

Fuente: Julio Genaro Pérez, Historia Electoral Dominicana. 1986. 
ELECCIONES PRESIDENCIALES 1848-2000 POR VOTOS INDIRECTOS

\begin{tabular}{|llrl|}
\hline Año & Candidato ganador & Votos & Opositor principal \\
1848 & Manuel Jiménes & 63 & Otros candidatos \\
1849 & Santiago Espaillat & 45 & Otros candidatos \\
1849 & Buenaventura Báez & 57 & Otros candidatos \\
1853 & Pedro Santana & 72 & \\
1859 & Pedro Santana & 124 & \\
1888 & Ulises Heureaux & 558 & \\
1892 & Ulises Heureaux & 393 & \\
1896 & Ulises Heureaux & 599 & José de Js. Alvarez \\
1899 & Juan I. Jiménes & 571 & Otros candidatos \\
1903 & Alejandro Woss y Gil & 509 & Otros candidatos \\
1904 & Carlos Morales L. & 413 & Otros candidatos \\
1908 & Ramón Cáceres & 578 & Otros candidatos \\
1912 & Eladio Victoria & 550 & Otros candidatos \\
1914 & Juan L. Jiménes & 337 & Horacio Vásquez \\
1924 & Horacio Vásquez & 210 & Fco J. Peynado \\
\hline
\end{tabular}

Fuente: Julio Genaro Campillo Pérez, Historia Electoral Dominicana. 1986.

hacer una tipología comprensiva como la que sigue a continuación.

Por las cifras del cuadro siguiente vemos que un número significativo de las elecciones dominicanas se han celebrado en contextos de fuerte efervescencia revolucionaria. En casi todos es-

Contexto de fuerte efervescencia revolucionaria

Contexto de relevo constitucionai

21

Contexto de intervención militar extranjera 
tos casos, los resultados de éstas sirvieron para coronar el triunfo alcanzado en los campos de batalla.

En el contexto de las dos ocupaciones militares hechas por los Estados Unidos al país se celebraron par de elecciones. Dada su cantidad, son fenómenos estadísticamente excepcionales.

Lo que más se destaca del pequeño cuadro anterior es que la mayoría de las elecciones dominicanas se han producido en un contexto de relevo constitucional, en el que constitución y las leyes imponían el relevo gubernamental. Siendo esto así se podría inferir que el país existe una vieja tradición electoral democrática, que en determinados momentos se ha visto obstruida por elecciones más bien irregulares, que han empañado lo que ha sido la regla. No obstante, una revisión a fondo del contexto en el que se han producido estas elecciones fuerza a refutar semejante conjetura.

Para entender el caso de las elecciones dominicanas que se han producido como consecuencia de las exigencias constitucionales es imprescindible subclasificar esta tipología en por lo menos 4 subcategorías como aparecen en el próximo cuadro:

Por un lado, el cuadro previo nos permite apreciar que en la República Dominicana no se produce ningún cambio de gobierno por la vía electoral, dentro del contexto de relevo constitucio-

\begin{tabular}{|l|l|}
\hline CONTEXTOS & $\begin{array}{l}\text { ELECCIONES } \\
\text { SEGÚN AÑOS }\end{array}$ \\
\hline $\begin{array}{l}\text { Relevo constitucional en medio } \\
\text { de una dictadura de partido }\end{array}$ & $1882 / 1884 / 1886$ \\
$\begin{array}{l}\text { Relevo constitucional en medio } \\
\text { de una dictadura personal }\end{array}$ & $1888 / 1892.1896 / 1934$ \\
$\begin{array}{l}\text { Relevo constitucional en medio } \\
\text { de fuertes restricciones a la oposición }\end{array}$ & $1938 / 1942 / 1947 / 1952 / 1957$ \\
Relevo constitucional en medio de & $1982 / 1986 / 1990 / 1994$ \\
competencia abierta entre rivales & $1996 / 2000$ \\
\hline
\end{tabular}


nal, sino hasta el año de 1882 , lo que es otra evidencia clave de la inestabilidad política que caracteriza la historia del país, especialmente en el siglo XIX.

El cuadro presente también pone de manifiesto que la gran mayoría de las elecciones dominicanas que se han celebrado en un contexto de relevo constitucional han sido en medio de situaciones de excepción política; bajo gobiernos que cuando no estaban dirigidos por jefes de estado francamente tiránicos, lo estaban por partidos que monopolizaban el gobierno y, no aceptaban auténticos opositores; o por gobernantes que sin ejercer el poder por medio de una dictadura personal, se las ingeniaban para organizar comicios electorales en medio de los cuales se coartaron los derechos de la oposición para que ésta no participara en esos certámenes electorales. En el primer caso estamos hablando de las elecciones celebradas bajo los gobiernos de Ulises Heureaux y Rafael Trujillo. En el segundo caso aludimos las elecciones que tuvieron lugar en el interregno 1880-1886, en las que fue decisiva políticamente, la dictadura del partido azul. En el tercer caso nos referimos, por supuesto, a las elecciones celebradas durante el primer ciclo de los doce años de Joaquín Balaguer.

El relevo constitucional en medio de competencia abierta entre rivales es un fenómeno extremadamente reciente en República Dominicana. Su primera manifestación data de 1982 y se ha mantenido hasta las elecciones del año 2000. Cabe, sin embargo, hacer todavía otros reparos imprescindibles a este análisis.

Es cierto que en las elecciones de 1982, 1986, 1990, 1994, 1996 y en las del año 2000 se produjo una competencia abierta entre rivales, hasta el momento mismo en que los electores concurrieron a las urnas. Empero, los resultados finales, en casi estos comicios fueron muy diferentes a los expresados en las urnas. Solo en 1982 y 1996 los electores decidieron con sus votos el partido ganador de las elecciones. En los otros casos, cuando no 
se impuso un triunfador, se hicieron negociaciones que culminaron, la mayoría tras profundas crisis políticas.

Los resultados de las elecciones del año 2000 se negociaron en medio de una breve crisis. Los resultados que favorecieron el triunfo del Partido Revolucionario Dominicano se validaron por dos hechos determinantes: 1) El Partido Reformista Social Cristiano se adelantó a reconocer el triunfo del Partido Revolucionario Dominicano, y 2) lo que obligó al Partido de la Liberación Dominicana a renunciar a participar en una segunda vuelta electoral. Las elecciones del año 2000 quedarán marcadas por el paro del escrutinio de los votos y por la negociación que se produjo a continuación para que se obviara el mandato legal que establecía que para acreditarle el triunfo a cualesquiera de los partidos contendores en la primera vuelta, éste debía completar un $50 \%$ más uno de los votos.

A pesar de sus negociaciones furtivas, el certamen electoral del año 2000 constituye un paradigma de apertura y participación, junto con los de 1982 y 1996. Indiscutiblemente que tiene muchos elementos que lo diferencian de la mayoría de los procesos precedentes. Entre ellos, uno harto significativo: una vez se pusieron de acuerdo los tres partidos contrincantes del certamen, no se produjo ningún tipo de impugnación de los resultados. De esta forma la transición entre el pasado y el nuevo gobierno pareció tan prolongada, que se debatió con insistencia en torno a reducir el tiempo de cambio de gobierno.

Se puede afirmar, sin lugar a dudas, que a pesar de que en el país se han celebrado 24 elecciones dentro de un contexto de relevo constitucional, apenas dos de ellas se han efectuado en medio de competencia abierta entre los rivales. Estas elecciones fueron las de 1982 y 1996 